\title{
A generalization of Sperner's theorem on compressed ideals
}

\author{
Lili $\mathrm{Mu} \quad$ Yi Wang * \\ School of Mathematical Sciences \\ Dalian University of Technology \\ Dalian, PR China \\ lly-mu@hotmail.com wangyi@dlut.edu.cn
}

Submitted: Dec 1, 2015; Accepted: Jul 27, 2016; Published: Aug 5,2016

Mathematics Subject Classifications: 05D05, 05A20

\begin{abstract}
Let $[n]=\{1,2, \ldots, n\}$ and $\mathscr{B}_{n}=\{A: A \subseteq[n]\}$. A family $\mathscr{A} \subseteq \mathscr{B}_{n}$ is a Sperner family if $A \nsubseteq B$ and $B \nsubseteq A$ for distinct $A, B \in \mathscr{A}$. Sperner's theorem states that the density of the largest Sperner family in $\mathscr{B}_{n}$ is $\left(\begin{array}{c}n \\ \lceil n / 2\rceil\end{array}\right) / 2^{n}$. The objective of this note is to show that the same holds if $\mathscr{B}_{n}$ is replaced by compressed ideals over $[n]$.
\end{abstract}

Keywords: Convex family; Sperner family; Ideal; Filter; Compressed ideal

\section{Introduction}

Let $\mathscr{B}_{n}$ be the poset of subsets of $[n]=\{1,2, \ldots, n\}$ ordered by inclusion. A family $\mathscr{A} \subseteq \mathscr{B}_{n}$ is a Sperner family if $A \nsubseteq B$ and $B \nsubseteq A$ for distinct $A, B \in \mathscr{A}$. A famous result due to Sperner [5] states that the density of the largest Sperner family in $\mathscr{B}_{n}$ is $\left(\begin{array}{c}n \\ {[n / 2\rceil}\end{array}\right) / 2^{n}$. Sperner's theorem is one of the central results in extremal finite set theory and it has many generalizations and extensions (see $[1,2]$ for instance).

For $\mathscr{P} \subseteq \mathscr{B}_{n}$, we say that $\mathscr{P}$ is a convex family if $A, B \in \mathscr{P}$ and $A \subseteq C \subseteq B$ imply that $C \in \mathscr{P}$. A family $\mathscr{I} \subseteq \mathscr{B}_{n}$ is an ideal if $A \in \mathscr{I}$ and $B \subseteq A$ imply $B \in \mathscr{I}$. Clearly, an ideal is a convex family. In [3, Conjecture 1.3], Frankl conjectured that the density of the largest Sperner family in any convex subfamily of $\mathscr{B}_{n}$ is at least $\left(\begin{array}{c}n \\ {[n / 2\rceil}\end{array}\right) / 2^{n}$.

Conjecture 1. For every convex family $\mathscr{P}$ over the set $[n]$, there exists a Sperner family $\mathscr{A} \subseteq \mathscr{P}$ such that

$$
|\mathscr{A}| /|\mathscr{P}| \geqslant\left(\begin{array}{c}
n \\
\lceil n / 2\rceil
\end{array}\right) / 2^{n} .
$$

*Supported by National Natural Science Foundation of China (No. 11371078). 
The conjecture seems difficult to prove and no progress was made in more than 30 years. Since no progress for the general case was made, it is quite natural to consider the special case of ideals. Here, we will restrict our research to the compressed ideals of $\mathscr{B}_{n}$. On $\mathscr{B}_{n}$ we consider the reverse lexicographic order $\preceq$, which is defined by $A \preceq A^{\prime}$ if $\max \left\{\left(A \cup A^{\prime}\right) \backslash\left(A \cap A^{\prime}\right)\right\} \in A^{\prime}$ or $A=A^{\prime}$ for $A, A^{\prime} \in \mathscr{B}_{n}$. For example, we have $\{3,4\} \preceq\{1,3,5\}$ and $\{3,5\} \preceq\{1,3,5\}$. Let $\mathcal{C}\left(m, \mathscr{B}_{n}\right)$ be the family of the first $m$ minimal elements of $\mathscr{B}_{n}$ with respect to $\preceq$. The family $\mathcal{C}\left(m, \mathscr{B}_{n}\right)$ is called compressed and the operation of exchanging an $m$-element family of $\mathscr{B}_{n}$ by $\mathcal{C}\left(m, \mathscr{B}_{n}\right)$ is called compression. Denote by $\mathscr{B}_{n}^{(k)}$ the collection of all $k$-subsets of $\mathscr{B}_{n}$. Similarly, we define $\mathcal{C}(\mathscr{F})$, where $\mathscr{F} \subseteq \mathscr{B}_{n}^{(k)}$, to be the first $|\mathscr{F}|$ elements of $\mathscr{B}_{n}^{(k)}$ with respect to $\preceq$. Here, we use the compress notation from $[1$, Ch. 7.5$]$ and $[2$, p. 41$]$. An ideal $\mathscr{I}$ is called a compressed ideal if $\mathcal{C}\left(\mathscr{I} \cap \mathscr{B}_{n}^{(k)}\right)=\mathscr{I} \cap \mathscr{B}_{n}^{(k)}$ for all $0 \leqslant k \leqslant n$. Clearly, $\mathscr{B}_{n}$ is a compressed ideal. In this paper, we will prove the following result.

Theorem 2. Let $\mathscr{I}$ be a compressed ideal in $\mathscr{B}_{n}$ and $\mathscr{A}$ the largest Sperner family in $\mathscr{I}$. Then

$$
|\mathscr{A}| /|\mathscr{I}| \geqslant\left(\begin{array}{c}
n \\
\lceil n / 2\rceil
\end{array}\right) / 2^{n} .
$$

\section{Proof of Theorem 2}

Let $\mathscr{A} \subseteq \mathscr{B}_{n}^{(k)}$ where $k<n$. Call

$$
\Delta \mathscr{A}=\left\{B \in \mathscr{B}_{n}^{(k-1)}: \exists A \in \mathscr{A}, B \subset A\right\}
$$

the shadow of $\mathscr{A}$ and

$$
\nabla \mathscr{A}=\left\{B \in \mathscr{B}_{n}^{(k+1)}: \exists A \in \mathscr{A}, A \subset B\right\}
$$

the shade of $\mathscr{A}$.

As usual, we let

$$
\left(\begin{array}{l}
x \\
k
\end{array}\right)=\frac{x(x-1) \cdots(x-k+1)}{k !},
$$

for $x \in \mathbb{R}^{+}$and $k \in \mathbb{Z}^{+}$.

To prove Theorem 2, we need two lemmas.

Lemma 3. [1] Let $\mathscr{F} \subseteq \mathscr{B}_{n}^{(k)}$. Then $|\Delta \mathscr{F}|>|\mathscr{F}|$ if $k>\lceil n / 2\rceil$ and $|\nabla \mathscr{F}|>|\mathscr{F}|$ if $k<\lfloor n / 2\rfloor$.

Lemma 4. [4] Let $\mathscr{F} \subseteq \mathscr{B}_{n}^{(k)}$. Then there is an $x \geqslant k$ such that $|\mathscr{F}|=\left(\begin{array}{l}x \\ k\end{array}\right)$ and $|\Delta \mathscr{F}| \geqslant\left(\begin{array}{c}x \\ k-1\end{array}\right)$.

Proof of Theorem 2. To simplify the notation, let us write

$$
T(n)=\left(\begin{array}{c}
n \\
\lceil n / 2\rceil
\end{array}\right) / 2^{n}
$$


It can be verified that $T(2 n-1)=T(2 n)$ and $T(2 n) / T(2 n+1)=(2 n+2) /(2 n+1)$. Hence we have

$$
T(1)=T(2)>T(3)=T(4)>\cdots>T(2 m-1)=T(2 m)>\cdots .
$$

We use induction on $n$. The case $n=1$ is trivial. So we proceed to the induction step. Let $\mathscr{I}$ be a compressed ideal in $\mathscr{B}_{n}$. Then $\mathscr{I}=\mathscr{I}_{1} \cup \mathscr{I}_{2}$, where $\mathscr{I}_{1}=\{A \in \mathscr{I}: n \notin A\}$ and $\mathscr{I}_{2}=\{A \in \mathscr{I}: n \in \mathscr{I}\}$. Denote by $\mathscr{I}_{2}(\bar{n})$ the collection of all sets $A \backslash\{n\}$, with $A \in \mathscr{I}_{2}$. Clearly, $\mathscr{I}_{1}$ and $\mathscr{I}_{2}(\bar{n})$ are compressed ideals in $\mathscr{B}_{n-1}$. We therefore use the induction hypothesis for $\mathscr{B}_{n-1}$, assuming that there exists the largest Sperner families $\mathscr{A}_{1} \subseteq \mathscr{I}_{1}$ and $\mathscr{A}_{2}(\bar{n}) \subseteq \mathscr{I}_{2}(\bar{n})$ such that

$$
\frac{\left|\mathscr{A}_{1}\right|}{\left|\mathscr{I}_{1}\right|} \geqslant T(n-1), \quad \frac{\left|\mathscr{A}_{2}(\bar{n})\right|}{\left|\mathscr{I}_{2}(\bar{n})\right|} \geqslant T(n-1) .
$$

Let $\mathscr{A}_{2}=\left\{A \cup\{n\}: A \in \mathscr{A}_{2}(\bar{n})\right\}$. Then $\mathscr{A}_{2}$ is the largest Sperner family in $\mathscr{I}_{2}$ and

$$
\frac{\left|\mathscr{A}_{2}\right|}{\left|\mathscr{I}_{2}\right|}=\frac{\left|\mathscr{A}_{2}(\bar{n})\right|}{\left|\mathscr{I}_{2}(\bar{n})\right|} \geqslant T(n-1) \geqslant T(n) .
$$

Denote by $\mathscr{I}_{i}^{(k)}$ the collection of all sets $\mathscr{I}_{i}$ which occur in $\mathscr{B}_{n}^{(k)}$, and $\mathscr{A}_{i}^{(k)}$ the collection of all sets $\mathscr{A}_{i}$ which occur in $\mathscr{I}_{i}^{(k)}$ for $i=1,2$. Let $s=\min \left\{k: \mathscr{A}_{1}^{(k)} \neq \emptyset\right\}$ and $r=$ $\max \left\{k: \mathscr{A}_{2}^{(k)} \neq \emptyset\right\}$. Then we have $\mathscr{I}_{1}^{(r)}=\mathscr{B}_{n-1}^{(r)}$ by the definition of compressed ideal. Hence $\mathscr{I}_{1}$ can be written as

$$
\mathscr{I}_{1}=\bigcup_{k=0}^{r} \mathscr{B}_{n-1}^{(k)} \cup \bigcup_{k>r} \mathscr{I}_{1}^{(k)}
$$

We now prove that $r \leqslant\lceil n / 2\rceil$. Note that $\mathscr{A}_{2}^{(r)} \subseteq \mathscr{B}_{n}^{(r)}$ and then $A_{2}^{(r)}(\bar{n}) \subseteq \mathscr{B}_{n-1}^{(r-1)}$. Hence by Lemma 3 , if $r-1>\lceil(n-1) / 2\rceil$, then

$$
\left|\Delta\left(\mathscr{A}_{2}^{(r)}(\bar{n})\right)\right|>\left|\mathscr{A}_{2}^{(r)}(\bar{n})\right| .
$$

Replacing $\mathscr{A}_{2}^{(r)}(\bar{n})$ by $\Delta\left(\mathscr{A}_{2}^{(r)}(\bar{n})\right)$, we obtain a larger Sperner family than $\mathscr{A}_{2}(\bar{n})$ in $\mathscr{I}_{2}(\bar{n})$. Thus $r-1 \leqslant\lceil(n-1) / 2\rceil$, i.e., $r \leqslant\lceil n / 2\rceil$.

In the following, we show that there is the largest Sperner family $\mathscr{A}$ in $\mathscr{I}$ such that (1) holds. We distinguish two cases.

Case 1: we consider the case that $n$ is even. Let $n=2 m$. Then $r \leqslant m$. We show that $s \geqslant r$. Assume that $s<r$. Let

$$
\overline{\mathscr{A}}_{1}=\left(\mathscr{A}_{1} \backslash\left\{\mathscr{A}_{1} \cap \mathscr{I}_{1}^{(s)}\right\}\right) \cup\left(\nabla^{(r)}\left(\mathscr{A}_{1} \cap \mathscr{I}_{1}^{(s)}\right)\right)
$$

where

$$
\nabla^{(r)}\left(\mathscr{A}_{1} \cap \mathscr{I}_{1}^{(s)}\right)=\left\{A \in \mathscr{B}_{2 m-1}^{(r)}: \exists B \in \mathscr{A}_{1} \cap \mathscr{I}_{1}^{(s)}, A \supset B\right\}
$$


By (4), $\nabla^{(r)}\left(\mathscr{A}_{1} \cap \mathscr{I}_{1}^{(s)}\right) \subset \mathscr{I}_{1}$ and $\overline{\mathscr{A}}_{1}$ is still a Sperner family in $\mathscr{I}_{1}$. By Lemma 3 ,

$$
\left|\nabla^{(r)}\left(\mathscr{A}_{1} \cap \mathscr{I}_{1}^{(s)}\right)\right|>\left|\mathscr{A}_{1} \cap \mathscr{I}_{1}^{(s)}\right|
$$

so that $\left|\overline{\mathscr{A}}_{1}\right|>\left|\mathscr{A}_{1}\right|$ which contradicts the maximality of $\mathscr{A}_{1}$ in $\mathscr{I}_{1}$. Hence we have $s \geqslant r$, which means that $\mathscr{A}_{1} \cup \mathscr{A}_{2}$ is still a Sperner family in $\mathscr{I}$. Hence by (2) and (3), we have

$$
\frac{\left|\mathscr{A}_{1} \cup \mathscr{A}_{2}\right|}{|\mathscr{I}|}=\frac{\left|\mathscr{A}_{1}\right|+\left|\mathscr{A}_{2}\right|}{\left|\mathscr{I}_{1}\right|+\left|\mathscr{I}_{2}\right|} \geqslant T(2 m-1)=T(2 m)
$$

and thus $\mathscr{A}=\mathscr{A}_{1} \cup \mathscr{A}_{2}$ is the family as desired.

Case 2: we consider the case that $n$ is odd. Let $n=2 m+1$. Then $r \leqslant m+1$. If $r<m+1$, by (4) we similarly have $s \geqslant r$, and thus $\mathscr{A}=\mathscr{A}_{1} \cup \mathscr{A}_{2}$ is the family as desired. If $r=m+1$, then

$$
\mathscr{I}_{1}=\bigcup_{k=0}^{m+1} \mathscr{B}_{2 m}^{(k)} \cup \bigcup_{k>m+1} \mathscr{I}_{1}^{(k)}
$$

and $\mathscr{A}_{1}=\mathscr{B}_{2 m}^{(m)}$. However $\mathscr{B}_{2 m}^{(m)} \cup \mathscr{A}_{2}$ is no longer a Sperner family. Let

$$
\overline{\mathscr{A}}_{2}=\left(\mathscr{A}_{2} \backslash\left\{\mathscr{A}_{2}^{(m+1)}\right\}\right) \cup\left(\Delta_{1}\left(\mathscr{A}_{2}^{(m+1)}\right)\right),
$$

where $\Delta_{1}\left(\mathscr{A}_{2}^{(m+1)}\right)$ is the shadow of $\mathscr{A}_{2}^{(m+1)}$ in $\mathscr{I}_{2}$, i.e.,

$$
\Delta_{1}\left(\mathscr{A}_{2}^{(m+1)}\right) \triangleq \Delta\left(\mathscr{A}_{2}^{(m+1)}(\overline{2 m+1})\right) \cup\{2 m+1\} .
$$

Then $\overline{\mathscr{A}}_{2}$ is still a Sperner family in $\mathscr{I}_{2}$. Moreover, $\mathscr{B}_{2 m}^{(m)} \cup \overline{\mathscr{A}}_{2}$ is also a Sperner family in $\mathscr{I}$. We then show that

$$
\frac{\left|\mathscr{B}_{2 m}^{(m)} \cup \overline{\mathscr{A}}_{2}\right|}{\left|\mathscr{I}_{1}\right|+\left|\mathscr{I}_{2}\right|} \geqslant T(2 m+1) .
$$

We first claim that

$$
\left(\begin{array}{c}
2 m \\
m
\end{array}\right) \geqslant(2 m+1)\left|\mathscr{A}_{2}\right|-(2 m+2)\left|\overline{\mathscr{A}}_{2}\right|
$$

Note that

$$
\left|\mathscr{A}_{2}\right|=\left|\mathscr{A}_{2}^{(m+1)}\right|+\sum_{i<m+1}\left|\mathscr{A}_{2}^{(i)}\right|
$$

and

$$
\left|\overline{\mathscr{A}}_{2}\right|=\left|\Delta_{1}\left(\mathscr{A}_{2}^{(m+1)}\right)\right|+\sum_{i<m+1}\left|\mathscr{A}_{2}^{(i)}\right|
$$


Hence we get that

$$
(2 m+1)\left|\mathscr{A}_{2}\right|-(2 m+2)\left|\overline{\mathscr{A}}_{2}\right| \leqslant(2 m+1)\left|\mathscr{A}_{2}^{(m+1)}\right|-(2 m+2)\left|\Delta_{1}\left(\mathscr{A}_{2}^{(m+1)}\right)\right| .
$$

So, to show that (6) is correct, it suffices to show that the following inequality is correct.

$$
\left(\begin{array}{c}
2 m \\
m
\end{array}\right) \geqslant(2 m+1)\left|\mathscr{A}_{2}^{(m+1)}\right|-(2 m+2)\left|\Delta_{1}\left(\mathscr{A}_{2}^{(m+1)}\right)\right| .
$$

Actually,

$$
\mathscr{A}_{2}^{(m+1)}(\overline{2 m+1}) \subseteq \mathscr{I}_{2}^{(m+1)}(\overline{2 m+1}) \subseteq \mathscr{B}_{2 m}^{(m)}
$$

Suppose that

$$
\left|\mathscr{A}_{2}^{(m+1)}(\overline{2 m+1})\right|=\left(\begin{array}{c}
x \\
m
\end{array}\right),
$$

where $m \leqslant x \leqslant 2 m$. Then by Lemma 4 , we have

$$
\left|\Delta_{1}\left(\mathscr{A}_{2}^{(m+1)}\right)\right|=\left|\Delta\left(\mathscr{A}_{2}^{(m+1)}(\overline{2 m+1})\right)\right| \geqslant\left(\begin{array}{c}
x \\
m-1
\end{array}\right) .
$$

Thus

$$
\begin{aligned}
& (2 m+1)\left|\mathscr{A}_{2}^{(m+1)}\right|-(2 m+2)\left|\Delta_{1}\left(\mathscr{A}_{2}^{(m+1)}\right)\right| \\
\leqslant & (2 m+1)\left(\begin{array}{c}
x \\
m
\end{array}\right)-(2 m+2)\left(\begin{array}{c}
x \\
m-1
\end{array}\right) \\
\leqslant & \left((2 m+1) \frac{x-m+1}{m}-(2 m+2)\right)\left(\begin{array}{c}
x \\
m-1
\end{array}\right) \\
\leqslant & \left((2 m+1) \frac{2 m-m+1}{m}-(2 m+2)\right)\left(\begin{array}{c}
2 m \\
m-1
\end{array}\right) \\
= & \frac{m+1}{m}\left(\begin{array}{c}
2 m \\
m-1
\end{array}\right) \\
= & \left(\begin{array}{c}
2 m \\
m
\end{array}\right) .
\end{aligned}
$$

This completes the proof of (7).

Then we show that (5) can be derived from (6). Actually, we have

$$
\left|\overline{\mathscr{A}}_{2}\right|+\frac{1}{2 m+2}\left(\begin{array}{c}
2 m \\
m
\end{array}\right) \geqslant \frac{2 m+1}{2 m+2}\left|\mathscr{A}_{2}\right|=\frac{T(2 m+1)}{T(2 m)}\left|\mathscr{A}_{2}\right| \geqslant T(2 m+1)\left|\mathscr{I}_{2}\right| .
$$

Replace $T(2 m+1)$ by $\left(\begin{array}{c}2 m+1 \\ m\end{array}\right) / 2^{2 m+1}$ and rewrite (8) as

$$
2^{2 m+1}\left|\overline{\mathscr{A}}_{2}\right|+\frac{2^{2 m+1}}{2 m+2}\left(\begin{array}{c}
2 m \\
m
\end{array}\right) \geqslant\left(\begin{array}{c}
2 m+1 \\
m
\end{array}\right)\left|\mathscr{I}_{2}\right| .
$$


We thus obtain

$$
\frac{\left(\begin{array}{c}
2 m \\
m
\end{array}\right)+\left|\overline{\mathscr{A}}_{2}\right|}{2^{2 m}+\left|\mathscr{I}_{2}\right|} \geqslant \frac{\left(\begin{array}{c}
2 m+1 \\
m
\end{array}\right)}{2^{2 m+1}}=T(2 m+1)
$$

by using the identity

$$
\frac{2^{2 m+1}}{2 m+2}\left(\begin{array}{c}
2 m \\
m
\end{array}\right)=2^{2 m+1}\left(\begin{array}{c}
2 m \\
m
\end{array}\right)-2^{2 m}\left(\begin{array}{c}
2 m+1 \\
m
\end{array}\right) .
$$

Hence

$$
\frac{\left|\mathscr{B}_{2 m}^{(m)} \cup \overline{\mathscr{A}}_{2}\right|}{\left|\mathscr{I}_{1}\right|+\left|\mathscr{I}_{2}\right|} \geqslant \frac{\left(\begin{array}{c}
2 m \\
m
\end{array}\right)+\left|\overline{\mathscr{A}}_{2}\right|}{2^{2 m}+\left|\mathscr{I}_{2}\right|} \geqslant T(2 m+1),
$$

as required. This completes the proof of Theorem 2 .

\section{Remarks}

Let $\mathscr{I}$ be an ideal in $\mathscr{B}_{n}$. The sequence $f(\mathscr{I})=\left(f_{0}(\mathscr{I}), f_{1}(\mathscr{I}), \ldots, f_{t}(\mathscr{I})\right)$, with $f_{k}(\mathscr{I})=$ $\left|\mathscr{I} \cap \mathscr{B}_{n}^{(k)}\right|$, is called the profile of the ideal $\mathscr{I}$. It is known that there exists a compressed ideal $\mathscr{I}^{\prime}$ sharing the same profile with the ideal $\mathscr{I}$ in $\mathscr{B}_{n}$ (see [1, Theorem 8.2.1] for details). By Theorem 2, there exists the largest Sperner family $\mathscr{A}^{\prime}$ in compressed ideal $\mathscr{I}^{\prime}$ such that $\left|\mathscr{A}^{\prime}\right| /\left|\mathscr{I}^{\prime}\right| \geqslant\left(\begin{array}{c}n \\ \lceil n / 2\rceil\end{array}\right) / 2^{n}$. So, a key step to show that the ideal $\mathscr{I}$ satisfies Conjecture 1 should be to find the relationship between the largest Sperner family $\mathscr{A} \in \mathscr{I}$ and $\mathscr{A}^{\prime}$.

\section{Acknowledgements}

The authors thank the anonymous referee for his/her careful reading and helpful comments.

\section{References}

[1] I. Anderson. Combinatorics of Finite Sets. Clarendon Press, Oxford, 1987.

[2] K. Engel. Sperner Theory. Cambridge University Press, Cambridge, 1997.

[3] P. Frankl and J. Akiyama. Modern Combinatorics. Kyoritsu, Tokyo, 1987.

[4] L. Lovász. Combinatorial Problems and Exercises. North-Holland, Amsterdam, 1979.

[5] E. Sperner. Ein Satzüber Untermengen einer endlichen Menge. Math. Z., 27:544-548, 1928. 